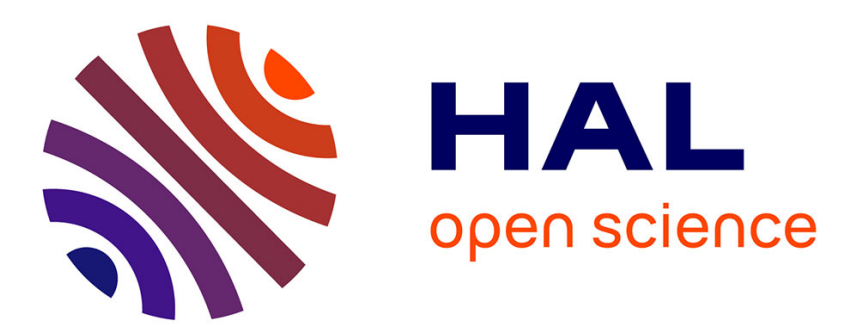

\title{
An Infrastructure-Free Slot Assignment Algorithm for Reliable Broadcast of Periodic Messages in Vehicular Ad hoc Networks
}

Mohamed Hadded, Anis Laouiti, Paul Mühlethaler, Leila Azouz Saidane

\section{- To cite this version:}

Mohamed Hadded, Anis Laouiti, Paul Mühlethaler, Leila Azouz Saidane. An Infrastructure-Free Slot Assignment Algorithm for Reliable Broadcast of Periodic Messages in Vehicular Ad hoc Networks. VTC Fall 2016, Sep 2016, Montréal Canada. hal-01379216

\author{
HAL Id: hal-01379216 \\ https://hal.science/hal-01379216
}

Submitted on 11 Oct 2016

HAL is a multi-disciplinary open access archive for the deposit and dissemination of scientific research documents, whether they are published or not. The documents may come from teaching and research institutions in France or abroad, or from public or private research centers.
L'archive ouverte pluridisciplinaire HAL, est destinée au dépôt et à la diffusion de documents scientifiques de niveau recherche, publiés ou non, émanant des établissements d'enseignement et de recherche français ou étrangers, des laboratoires publics ou privés.

$$
\text { Copyright }
$$




\title{
An Infrastructure-Free Slot Assignment Algorithm for Reliable Broadcast of Periodic Messages in Vehicular Ad hoc Networks
}

\author{
Mohamed Hadded ${ }^{* \dagger}$, Anis Laouiti*, Paul Muhlethaler ${ }^{\dagger}$, Leila Azouz Saidane ${ }^{\ddagger}$ \\ *TELECOM SudParis, CNRS Samovar, UMR 5157, France \\ ${ }^{\dagger}$ INRIA, BP 105. 78153 Le Chesnay Cedex, Paris-Rocquencourt, France \\ ${ }^{\ddagger}$ RAMSIS Team, CRISTAL Laboratory, 2010 Campus University, Manouba, Tunisia \\ \{mohamed.hadded, anis.laouiti\}@it-sudparis.eu, paul.muhlethaler@inria.fr, leila.saidane@ensi.rnu.tn
}

\begin{abstract}
A Vehicular Ad-Hoc NETwork (VANET) consists of a set of vehicles moving along roads, which can communicate with each other through ad hoc wireless devices. VANETs have attracted a great deal of attention in the research community in recent years, with the main focus being on their safety applications. One of the major challenges of vehicular networks is designing an efficient Medium Access Control (MAC) protocol which can cope with the hidden node problem, the high speed of the nodes, the frequent changes in topology, the lack of an infrastructure, and various $\mathrm{QoS}$ requirements. Motivated by this observation, we present a fully distributed and location-based TDMA scheduling scheme for VANETs, named DTMAC. The main goal of this work is to propose a MAC protocol that can provide a reliable broadcast service with bounded access delay, while reducing access collisions and merging collisions with various vehicle densities without having to use expensive and complex spectrum mechanisms such as CDMA or OFDMA. An analytical model of the average access collision probability has been derived, which can be used to evaluate the performance of DTMAC and validate the simulation results under different traffic conditions. The simulation results reveal that DTMAC significantly outperforms VeMAC in terms of transmission collisions and broadcast coverage.
\end{abstract}

Keywords-VANET, MAC Protocol, Collision-free, TDMA, Fully Distributed, Scheduling Scheme.

\section{INTRODUCTION}

The continuing increase in road traffic accidents throughout the world has motivated the development of Intelligent Transportation Systems (ITS) and other applications to improve road safety and driving comfort. A communication network, called a Vehicular Ad-hoc NETwork (VANET), in which the vehicles are equipped with wireless devices has been developed to make these applications feasible. In a VANET, communications can either be Vehicle-to-Vehicle (V2V) or Vehicle-to-Infrastructure (V2I) [1]. Based on these two types of communications, a VANET can support a wide range of applications for safety (such as dangerous situation detection), for infotainment (such as Internet access and data exchange) and for traffic management (such as vehicle traffic optimization).

Since safety applications in VANETs have stringent QoS requirements, an efficient Medium Access Control (MAC) protocol that can provide a broadcast service with bounded access delays and minimum transmission collision is required. Recently, MAC protocols, notably those that are based on the TDMA technique, have been used to enable multiple vehicles to use the same frequency channel without interfering with other vehicles' transmissions [1]. The TDMA principle consists in allocating the bandwidth to the vehicles by dividing the time into different frames and each frame is divided into several time slots. Each vehicle can access the channel during its dedicated time slot to send data messages, while it can only receive during the time slots reserved for other vehicles. However, many issues arise due to the high vehicle mobility in VANETs which can affect the performance of these protocols. Therefore, the scheduling mechanisms in TDMA-based MAC protocols should take into consideration the mobility features of VANETs. In this paper, we propose a completely Distributed and infrastructure free TDMA-based MAC protocol (DTMAC) which exploits the linear feature of topologies in VANETs. DTMAC uses vehicular location information to help the vehicles access the channel in an efficient way solve the collision problem caused by the high mobility of nodes and reduce the access delay.

The rest of the paper is organized as follows. In Section 2, we review related work. Section 3 describes the system models and presents the TDMA problems that may occur in a fully distributed VANET due to the high mobility of nodes. Section 4 describes our TDMA-based MAC protocol, called DTMAC and how it solves the hidden node problem without having to use complex broadband mechanisms such as FDMA or CDMA. An analytical model for the average access collision probability is presented in Section 5. Section 6 presents the simulation results and the performance evaluation. Finally, conclusions and future work are reported in Section 7.

\section{RELATED WORK}

MAC protocols generally fall into one of two broad categories: contention-based and contention-free [2]. In contentionbased protocols, each node can try to access the channel when it has data to transmit using the carrier sensing mechanism [3]. The IEEE 802.11p [4], which is the emerging standard deployed to enable vehicular communication, is a Contentionbased MAC protocol, using a priority-based access scheme that employs both Enhanced Distributed Channel Access (EDCA) and Carrier Sense Multiple Access with Collision Avoidance (CSMA/CA) mechanisms [5]. Since the IEEE 802.11p standard is a Contention-based MAC, it cannot provide a reliable broadcast mechanism with bounded communication 
delay. This disadvantage is particularly detrimental in VANETs which are specifically designed to improve road safety.

In Contention-free MAC protocols, only one vehicle can access the channel at any given time and a given neighborhood. Therefore, these protocols provide collision-free transmission with bounded access delay for real-time applications. In [6], the authors propose the Dedicated Multi-channel MAC (DMMAC) protocol which is an alternative to the IEEE 802.11p standard. DMMAC has an adaptive broadcasting mechanism providing collision-free and delay-bounded transmissions for safety applications under various traffic conditions. The DMMAC architecture is similar to IEEE $802.11 \mathrm{p}$ with the difference that, the $\mathrm{CCH}$ Interval is divided into an Adaptive Broadcast Frame (ABF) and a Contention-based Reservation Period (CRP). The ABF period consists of time slots, and each time slot is dynamically reserved by a vehicle as its Basic Channel $(\mathrm{BCH})$ for collision-free delivery of safety messages or other control messages. DMMAC implements a dynamic TDMA mechanism for $\mathrm{BCH}$ reservation based on the distributed access technique R-ALOHA (Reliable-ALOHA [7]). The length of the ABF is not uniform over the entire network. Each vehicle dynamically adjusts its ABF length according to its neighbors. In [8], Omar et al. developed and evaluated a contention-free multi-channel MAC protocol proposed for VANETs. In contrast to DMMAC, VeMAC is completely contention-free. This protocol supports efficient one-hop and multi-hop broadcast services on the control channel without the hidden terminal problem caused by node mobility. These broadcast services are presented in [9] for ADHOC MAC. VeMAC reduces the merging collision rate by assigning disjoint sets of time slots to vehicles moving in opposite directions (Left, Right) and to Road Side Units (RSUs). An efficient MAC approach called, ATSA [10] which is an improvement of the previously proposed MAC protocol based on VeMAC is named the Decentralized Adaptive TDMA Scheduling Strategy DATS [11]. Like VeMAC, ATSA divides the frame into two sets of time slots, Left and Right. However in ATSA, when a vehicle accesses the network, it chooses a frame length and competes for one of the time slots available for its direction. Moreover, the frame length is dynamically doubled or shortened based on the binary tree algorithm, and the ratio of two slot sets is adjusted to decrease the probability of transmission collisions. Dang et al. [12] developed and evaluated a Hybrid Efficient and Reliable MAC for Vehicular Ad hoc Networks, called HER-MAC, which is similar to the DMMAC protocol. The goal of this research work is to develop a contention-free Multichannel MAC protocol with an adaptive broadcasting algorithm, which improves data transfer rates for non-safety applications while guaranteeing timely delivery for safety applications in highway scenarios. The architecture and the operation of HER-MAC are similar to DMMAC, differing in that the CRP period is used by a vehicle to reserve a time slot during the $\mathrm{ABF}$ period or to exchange a 3-way WSA/RFS (WAVE Service Announcement/Request For Service) handshake. In fact, if a vehicle wishes to exchange non-safety messages, it has to broadcast the WSA during the CRP period to reserve a time slot on a certain SCH. Then, when a vehicle decides to use the service, it sends the RFS to the service provider which will confirm it with an ACK message. On receiving the ACK packet, the vehicles can start exchanging non-safety messages without any risk of collisions with messages from their neighboring vehicles.

The design of distributed TDMA-based MAC protocols for VANETs should take into account mobility (i.e., the slot scheduling mechanism should be periodically aware of the neighbors' slot allocations), scalability (i.e., they should scale under different traffic load conditions), and fairness (i.e., all the vehicles should have equal access to the medium during a fixed time interval) [1]. In this paper, a distributed and infrastructure free TDMA based MAC protocol (DTMAC) is proposed to address the above problems. In DTMAC, the road is divided into small fixed areas in which the time slots will be reused between them in such a way any vehicles in different adjacent areas may access the channel at the same time without any interference occurring.

\section{SYSTEM MODEL AND TDMA PROBLEM STATEMENT}

\section{A. System Model}

A VANET in a highway scenario consists of a set of vehicles moving in opposite directions and under varying traffic conditions (speed, density). DTMAC is based on the assumption that each vehicle in a VANET is equipped with a GPS (Global Positioning System) or a GALLILEO receiver that also allows it to obtain an accurate real-time three-dimensional geographic position (latitude, longitude and altitude), speed and exact time. Moreover, synchronization between vehicles may be performed by using GPS timing information. Each road is divided into small fixed areas (see Figure 2). Note that the area size depends on the transmission range of the vehicles (around $300 \mathrm{~m}$ ). Moreover, we assume that the vehicles are equipped with digital maps to determine which area they are in. In the following, we detail the slot scheduling mechanism in DTMAC and we show how this protocol can provide an efficient time slot utilization for the participating vehicles, while minimizing transmission collisions caused by the hidden node problem.

\section{B. TDMA Problem Statement}

When a distributed scheme is used to allocate a time slot, two types of collision can occur [13]: access collision between vehicles trying to access the same available time slots, and merging collisions between vehicles using the same time slots. When the traffic density is high, the rate of access and merging collisions will increase rapidly, which will lead to inefficient channel utilization and high access delay for safety applications. An access collision problem occurs when two or more vehicles within the same two-hop neighborhood set attempt to access the same available time slot. This problem is likely to happen when a distributed scheme is used. On the other hand, merging collisions occur when two vehicles in different two-hop sets accessing the same time slot become members of the same two-hop set due to changes in their position. Generally, in VANETs, merging collisions are likely to occur in the following cases:

- Vehicles moving at different speeds.

- Vehicles moving in opposite directions.

- $\quad$ There are RSUs installed along the road. 


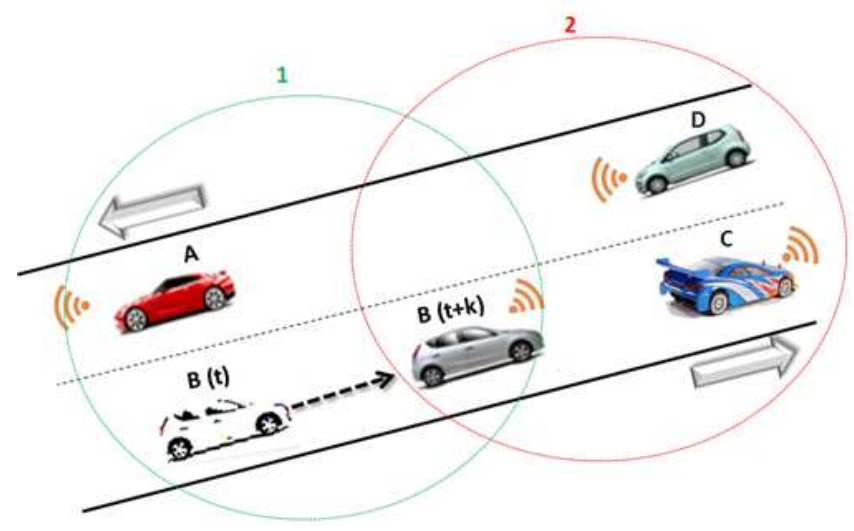

Fig. 1. Merging collision problem.

Figure 1 shows an example of the second case of the merging collision problem, when vehicle $\mathrm{B}$ in the first twohop set moving in the opposite direction to vehicle $\mathrm{E}$ in the second two-hop set is using the same time slot as B. Since $\mathrm{B}$ and $\mathrm{E}$ become members of the same two-hop set at instant $(t+k)$, a collision occurs at vehicle D.

\section{DISTRIBUTED AND INFRASTRUCTURE FREE TDMA-BASED MAC PROTOCOL}

\section{A. DTMAC Preliminaries}

We propose a completely distributed and infrastructure free TDMA scheduling scheme which exploits the linear feature of VANET topologies. The vehicles movements in a highway environment are linear due to the fact that their movements are constrained by the road topology. Our scheduling mechanism is also based on the assumption that each road is divided into $N$ small fixed areas, denoted by $x_{i}, i=1, \ldots, N$ (see Figure 2). Area IDs can be easily derived using map and GPS Information.

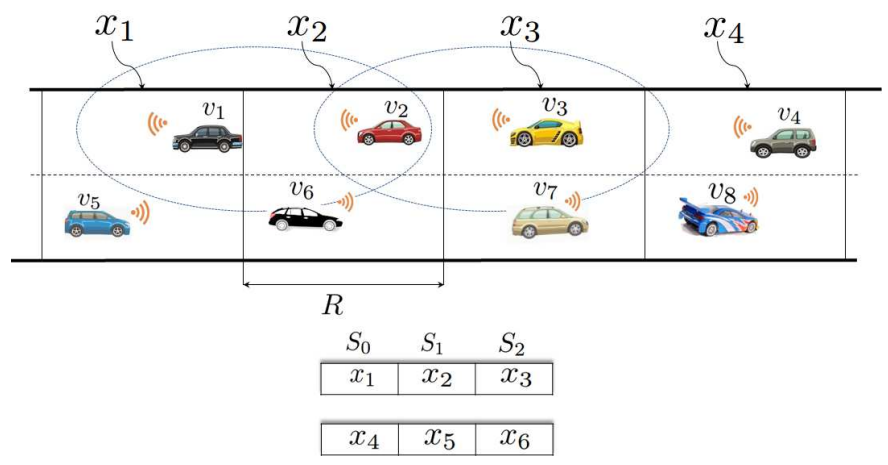

Fig. 2. TDMA slots scheduling principle.

The time slots in each TDMA frame are partitioned into three sets $S_{0}, S_{1}$ and $S_{2}$ associated with vehicles in three contiguous areas: $x_{i}, x_{i+1}$ and $x_{i+2}$, respectively (see Figure 2). Each frame consists of a constant number of time slots, denoted by $\tau$ and each time slot is of a fixed time duration, denoted by $s$. Each vehicle can detect the start time of each frame as well as the start time of a time slot. In the VANET studied, all the vehicles are equipped with a Global
Positioning System (GPS) and thus the one-Pulse-Per-Second (1PPS) signal that a GPS receiver gets from GPS satellites can be used for slot synchronization.

To prevent collisions on the transmission channel, our TDMA scheduling mechanism requires that every packet transmitted by any vehicle must contain additional information, called Frame Information (FI). The FI consists of a set of ID Fields (IDFs) of size equal to the number of time slots per frame, $\tau$. Each IDF is dedicated to the corresponding time slot of a frame. The basic FI structure is shown in Figure 3. Each time slot is dynamically reserved by an active vehicle (the vehicle whose communication device is transmitting) for collision-free delivery of safety messages or other control messages. The VC_ID field contains the ID of the vehicle that is accessing this slot. Each vehicle is identified by its MAC address. The SLT_STS field contains the status of each slot which indicates whether the slot is Idle, Busy or in Collision. Finally, the PKT_TYP field indicates the type of packet transmitted by the vehicle, i.e. periodic information or event-driven safety messages.

\section{B. TDMA slot scheduling mechanism}

Our distributed TDMA scheduling mechanism uses vehicles location and slot reuse concept to ensure that vehicles in adjacent areas have collision-free schedule. The channel time is partitioned into frames and each frame is further partitioned into three sets of time slots $S_{0}, S_{1}$, and $S_{2}$ of size equal to $n 1, n 2$ and $n 3$, respectively. These sets are associated with vehicles moving in the areas $x_{i}, x_{i+1}$, and $x_{i+2}$, respectively. As shown in Figure 2, by dividing the time slots into three sets, vehicles $v_{1}$ and $v_{3}$ that are moving within the two areas $x_{1}$ and $x_{3}$, respectively, can not transmit simultaneously to vehicle $v_{2}$ because they are accessing disjoint sets of time slots. Therefore, our TDMA scheduling mechanism can decrease the collisions rate caused by the hidden node problem in VeMAC. In each area, the vehicles access the time slots associated to their locations with the same probability. In the rest of the paper, we adopt the following notations:

- $\quad S_{j}(v)$ : The set of time slots associated to the area in which the vehicle $v$ is traveling.

- $\quad N(v)$ : The set of neighbors ${ }^{1}$ of vehicle $v$ on the transmission channel.

Every active vehicle in the network should be allocated a fixed slot in the frame for safety messages or other control packet transmissions. It is obvious that a vehicle's slot cannot be used by any neighboring vehicles within the same area or in adjacent areas, otherwise collisions will occur. The goal of this work is to propose an efficient slot reuse algorithm without having to use expensive spectrum and complex broadband mechanisms such as FDMA or CDMA. In fact, the three subsets of time slots will be reused between neighboring areas in such a way no vehicle in different adjacent areas can access the channel at the same time, and thus no interference will occur.

Let us suppose that an active vehicle $v$ moving within the area $x_{i}$ needs to acquire a time slot on the transmission

\footnotetext{
${ }^{1}$ The set of neighbors is the set of vehicles that are moving within the same area.
} 


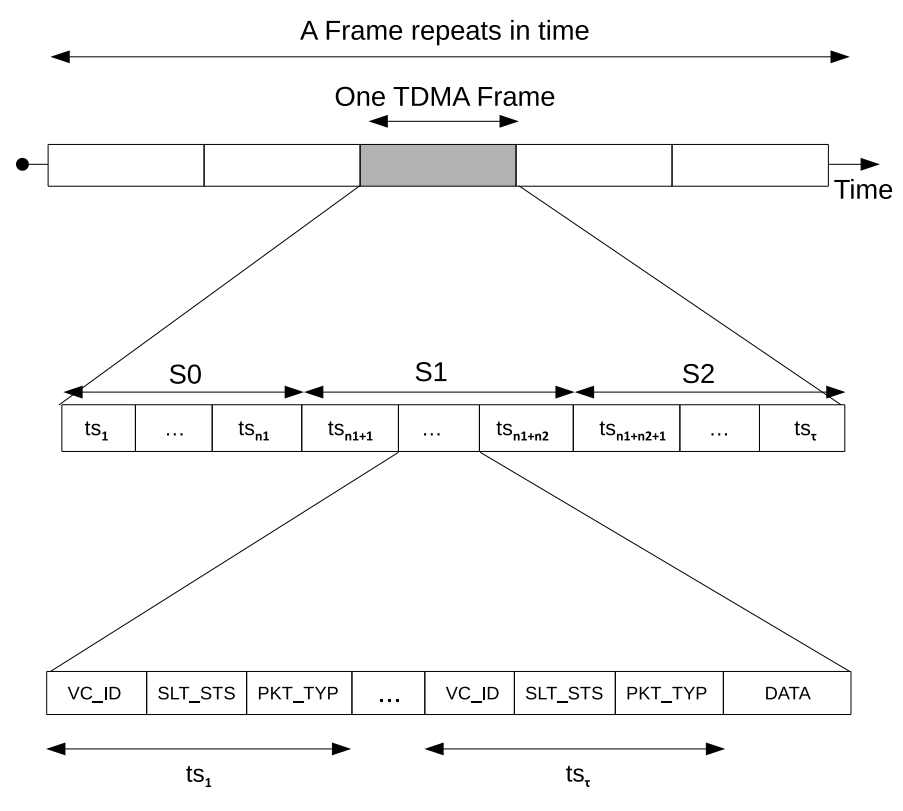

Fig. 3. Frame Information (FI) structure.

channel. Vehicle $v$ starts listening to the channel during the set $S_{j}(v)$ of time slots reserved for the area in which it is traveling, where $j=(i+2) \bmod 3$.

- Each vehicle that hears exactly one node transmission in a time slot reserved for its location, will set the status of the slot to "busy" and record the ID of the vehicle accessing the channel in this time slot in the corresponding VC_ID field.

- If a vehicle does not hear anything during a specific time slot, it will set its status to "free" in the FI.

- If a vehicle can not decode the data during a specific time slot, it will set its status to "collision" in the FI.

- When a vehicle A has sent data in a given slot, it looks in the field information of the next slots to discover whether its neighbors have correctly received its data. If a neighbor of A reports collision for this slot (in the FI) or even if this slot is reported to be "busy" but being sent by another node (say B in the VC_ID), A considers that its transmission has led to a collision ${ }^{2}$.

At the end of the frame the vehicle $v$ can determine the set $N(v)$ and the set of busy slots in $S_{j}(v)$ used by each vehicle $u \in N(v)$, denoted by $B(v)$. In order to avoid any collision problem, this set of time slots can not be used by any neighboring vehicles. Therefore, vehicle $v$ can determine the set of available time slots $F(v)$ and then attempts to select one of them at random, say time slot $k$.

Algorithm 1 outlines the details of how the frame information is built. In the algorithm, $i$ is the index of the area in which a vehicle is traveling. If no other vehicle moving in the

\footnotetext{
${ }^{2}$ Actually a node A considers that its transmission is a success if and only if all its neighbors report a success in the FI of their slots specifying that the data was sent by node A.
}

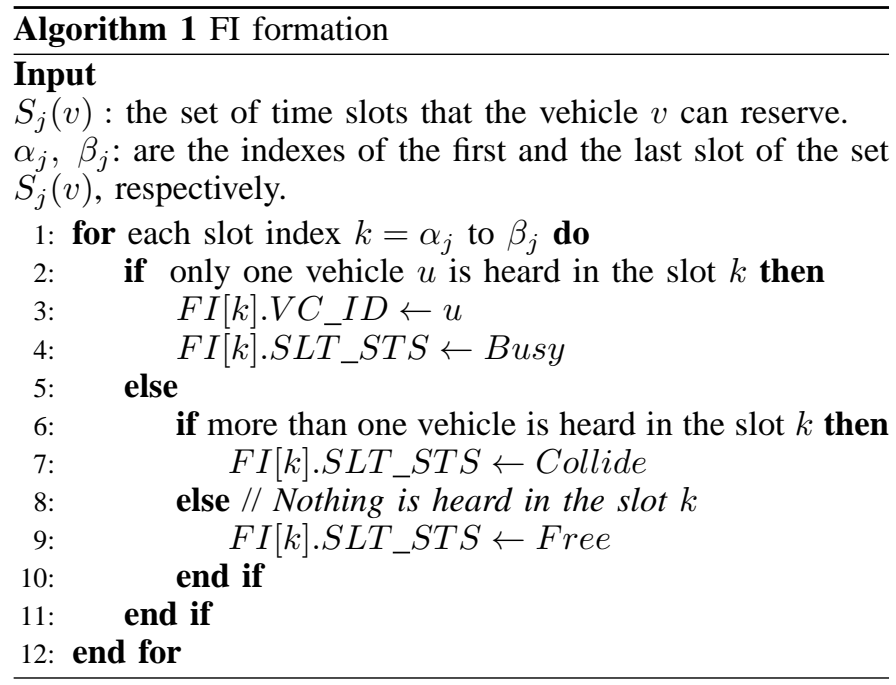

same area as vehicle $v$ attempts to acquire a time slot $k$, no access collision occurs. In this case, the attempt of vehicle $v$ is successful and all nodes $u \in N(v)$ add vehicle $v$ to their sets $N(u)$ and record that vehicle $v$ is using time slot $k$. However, if at least one node within the same area as vehicle $v$ accesses time slot $k$, then all the transmissions fail and the time slot $k$ is not acquired by any of the contending vehicles. In this case, vehicle $v$ will discover that its attempt was unsuccessful as soon as it receives a packet from any node $u \in N(v)$ indicating that vehicle $v \notin N(u)$. Vehicle $v$ then attempts to access one of the time slots in $F(v)$, and so on until all nodes $u \in N(v)$ indicate that node $v \in N(u)$ and announce that the time slot has been allocated to vehicle $v$. However, when an access collision occurs among the vehicles that are moving in the same area, the probability of access collision in the next reservation is increased since the choice of available slots will be restrained in the new set $F(v)$. In order to ensure channel access continuity, each vehicle should determine the expected available time slots on the set of time slots associated with the next area before leaving the area in which it is currently traveling. In fact, when a vehicle is using a given time slot in the set $S_{j}$, it should acquire an available time slot in the set $S_{(j+1) \bmod 3}$ as its future time slot before leaving its current area. Algorithm 2 outlines the details of the slot reservation mechanism. It is executed by each vehicle $v$ which needs to reserve a time slot.

\section{ACCESS COLLISION PROBABILITY}

In this section, we present a model to compute the average access collision probability. In this work, we assume that the VANET scenario taken into account is a two-way highway of length equal to $L$. We assume that every area of the road has a unique index number such as $1,2, \ldots, N$. The probability with which the vehicle in the $i-t h$ area decides to access the available $j-t h$ time slot reserved for its location is denoted by $p_{i j}$. For instance, the probability of the vehicle in the fourth area accessing the 7-th slot is denoted by $p_{47}$. First of all, we calculate the access collision probability when a vehicle tries to access an available time slot.

- $A_{i}$ : actual number of active vehicles in a given area $x_{i}$. 


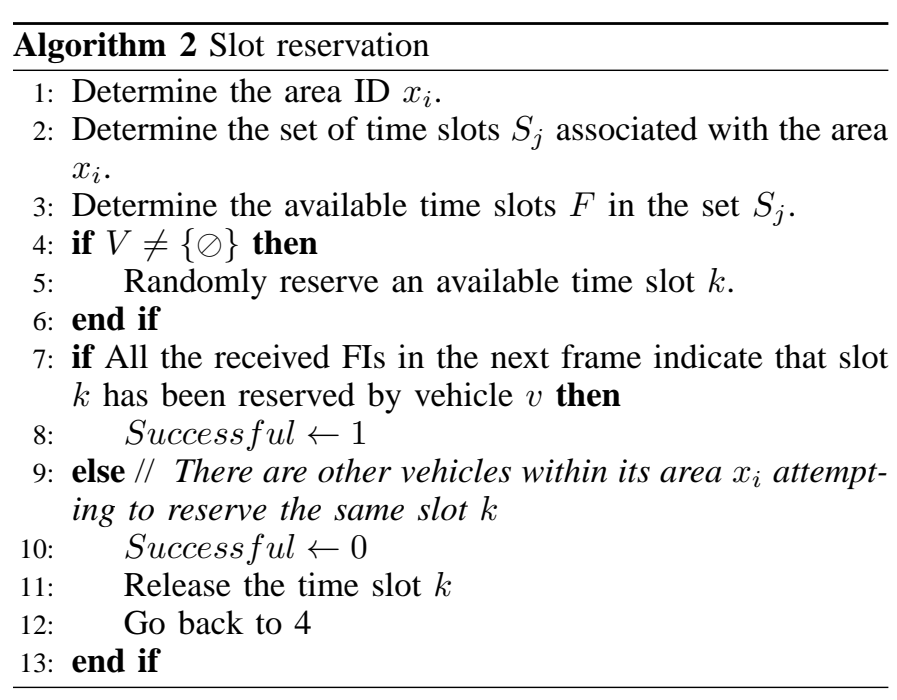

- $\quad P_{a c i}$ : the access collision probability of the vehicle in area $x_{i}$ accessing the channel.

- $\alpha_{i}, \beta_{i}$ : the indexes of the first and the last time slots reserved for the area $x_{i}$.

For DTMAC, the probability of accessing an available time slot $j$ by a contending vehicle $v$ in the area $i$ is $p_{i j}=$ $\frac{1}{\left(\beta_{i}-\alpha_{i}\right)-N_{s u c c_{i}}(v)}$, where $N_{\text {succ }_{i}}(v)$ is the number of vehicles in the area $i$ which have successfully acquired a time slot as derived from the framing information received by vehicle $v$. Therefore, the access collision probability of a vehicle in area $x_{1}$ can be evaluated as:

$$
\begin{gathered}
P_{a c 1}=1-P_{n a c 1} \\
P_{n a c 1}=\sum_{j=\alpha_{1}}^{\beta_{1}} p_{1 j} * \prod_{k=2}^{A_{1}}\left(1-p_{1 j}\right)
\end{gathered}
$$

where $P_{a c 1}$ denotes the access-collision probability in area $x_{1}$ and in a given time slot, while $P_{n a c 1}$ denotes the non accesscollision probability in area $x_{1}$ and in a given time slot.

Based on the above derivation, the expression of the total access collision probability of the vehicles in all locations can be given by:

$$
\begin{gathered}
P_{a c t}=1-P_{\text {nact }} \\
P_{\text {nact }}=\sum_{i=1}^{N} P_{\text {naci }}=\sum_{i=1}^{N} \sum_{j=\alpha_{i}}^{\beta_{i}} p_{i j} * \prod_{k=2}^{A_{i}}\left(1-p_{i j}\right)
\end{gathered}
$$

where, $P_{a c t}$ represents the total access-collision probability of the vehicle accessing the channel, $P_{\text {nact }}$ represents the total non access-collision probability of the vehicle accessing the channel.

$$
P_{\text {aver }-a c}=\frac{1}{N} * P_{a c t}
$$

$P_{\text {aver-ac }}$ represents the average access collision probability of the vehicle accessing the channel.

\section{SIMULATION RESULTS AND PERFORMANCE EVALUATION}

\section{A. Simulation Scenarios and Performance Metrics}

In our work, we have used VanetMobiSim [14] to generate the mobility pattern of vehicles. We simulate different traffic conditions by varying the speed deviation and the vehicles density. As shown in the Figure 4, we consider a VANET in a two-way highway scenario of size $2000 \mathrm{~m} \times 20 \mathrm{~m}$, where vehicles are moving along the highway in opposite directions. The parameters of VanetMobiSim consisted of the maximum number of vehicles, the starting and destination positions of each vehicle and the number of lanes per direction. During simulation time, each vehicle moves at a constant speed, and the number of vehicles on the highway remains constant. Then the traffic traces generated by VanetMobiSim were used in the ns 2.34 simulations. The simulation parameters used in our experiments are summarized in Table I.

We have used a parameter, called the area occupancy (AO) [8], equal to $\frac{N_{v} \times R}{L_{h} \times T_{s}}$ in a highway scenario, where $N_{v}$ is the total number of active vehicles, $R$ is the communication range, $L_{h}$ is the length of the highway, $T_{s}$ is the number of slots reserved for each area.

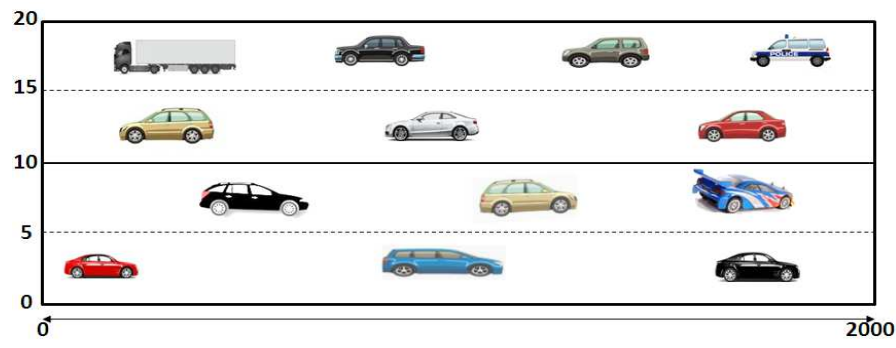

Fig. 4. VANET mobility scenario

TABLE I. Simulation parameters

\begin{tabular}{cc}
\hline Parameter & Value \\
\hline Highway length & $2 \mathrm{~km}$ \\
Lanes/direction & 2 \\
Vehicle speed & $120 \mathrm{~km} / \mathrm{h}$ \\
Speed standard deviation $(\sigma)$ & $30 \mathrm{~km} / \mathrm{h}$ \\
Transmission range & $300 \mathrm{~m}$ \\
Slots/frame & 100 \\
Slot duration & $0.001 \mathrm{~s}$ \\
Simulation time & $120 \mathrm{~s}$ \\
\hline
\end{tabular}

DTMAC is evaluated based on the following metrics:

1) The access collision rate: is defined as the average number of access collisions per slot per area.

2) The merging collision rate: is defined as the average number of merging collisions per slot per area.

3) The broadcast coverage ratio: is defined as the average of the total number of vehicles that successfully receive messages to the total number of vehicles within the communication range of the transmitter.

4) The packet loss rate: is defined as the average of the total number of vehicles that do not successfully 
receive messages to the total number of vehicles within the communication range of the transmitter.

\section{B. DTMAC performance evaluations}

The performance of DTMAC depends on the sizes of the three sets of time slots $n 1, n 2$ and $n 3$ that determine its behavior. An optimal tuning of these parameters can improve the QoS of DTMAC. For this, we evaluated several configurations in different speed scenarios (by varying the speed deviation $\sigma$ between 20,30 and $50 \mathrm{~km} / \mathrm{h}$ ) with different area occupancy values to find the optimal values of these parameters. Figure 5 shows the average access collision probability under various traffic conditions. The experiments were carried out for different values of $n 1, n 2$ and $n 3$. It is clear from these three figures that the first configuration when the three sets of time slots have the same size equal to $\tau / 3$, is the best configuration that minimizes the probability of access collision under different traffic conditions.

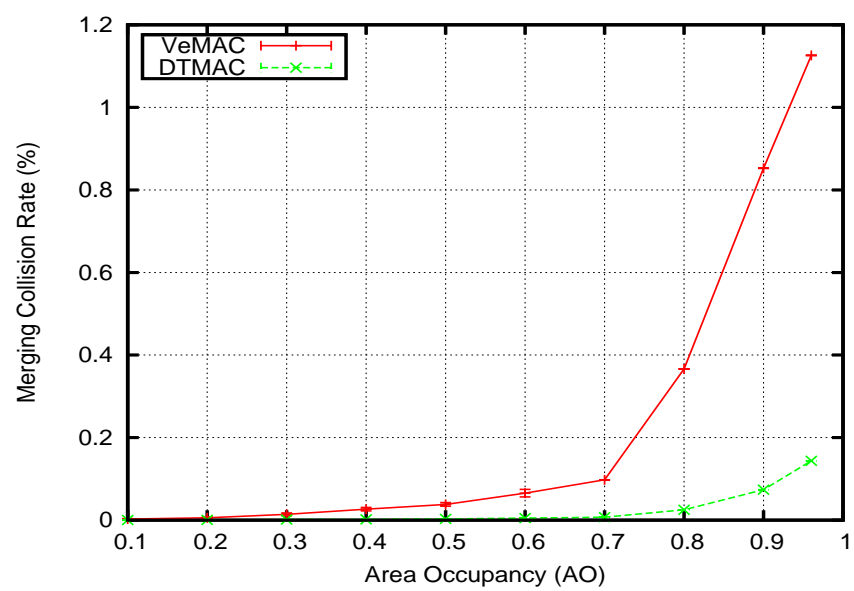

Fig. 6. The rate of merging collision.

Fig 6 shows the rate of merging collisions for DTMAC and VeMAC protocols when varying the Area Occupancy (AO). DTMAC $^{3}$ prevents more merging collisions than VeMAC even for a high $\mathrm{AO}$ since it assigns disjoint sets of time slots to vehicles moving in adjacent areas. However, in VeMAC, the vehicles that cannot access a time slot from the set of slots reserved for its direction, will attempt to access any available time slot reserved for vehicles moving in the opposite direction. Moreover, the available time slot sets are allocated by the contending vehicles without considering their speed deviations. Therefore merging-collisions occur frequently in VeMAC when traffic density is high as well as when vehicles driving toward each other and at high relative speeds. It should be notice that, in principle, the algorithm prevents any merging-collision for DTMAC.

Figure 7 shows the access collision rates of the two TDMA based MAC protocols. As shown in this Figure, DTMAC

\footnotetext{
${ }^{3}$ In principle, the DTMAC algorithm prevents any merging collision. However when errors at the physical layer lead to a reception error (the FI is not coherent with the transmission), a node may consider that its transmission is a collision even if it has been the sole transmitter within its zone in the slot. Thus, if this error is not on the first attempt of the node to acquire a slot, we consider that it is a merging collision.
}

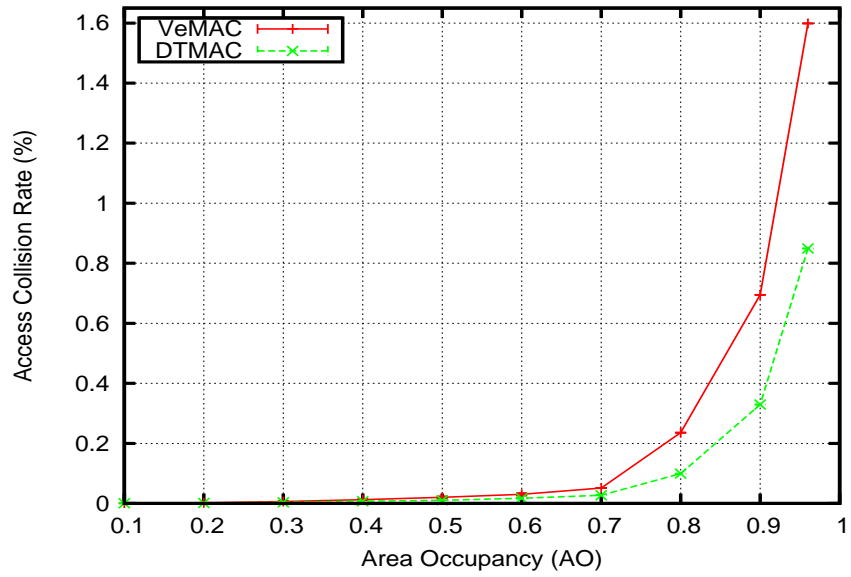

Fig. 7. The rate of access-collisions.

achieves a considerably smaller rate of access collisions than VeMAC, especially for a high AO $(\geq 0.7)$. For instance, at a $A O=0.96$, the DTMAC protocol achieves an access collision rate of $0.849 \%$, in contrast to VEMAC which shows a rate of $1.598 \%$ (i.e. approximately $88.22 \%$ higher than DTMAC). These results can be explained by the fact that VeMAC has achieved a higher rate of merging collision compared to DTMAC. Indeed, upon detection of merging-collisions, the nodes in collision should release their time slots and request new ones, which can reproduce access-collisions.

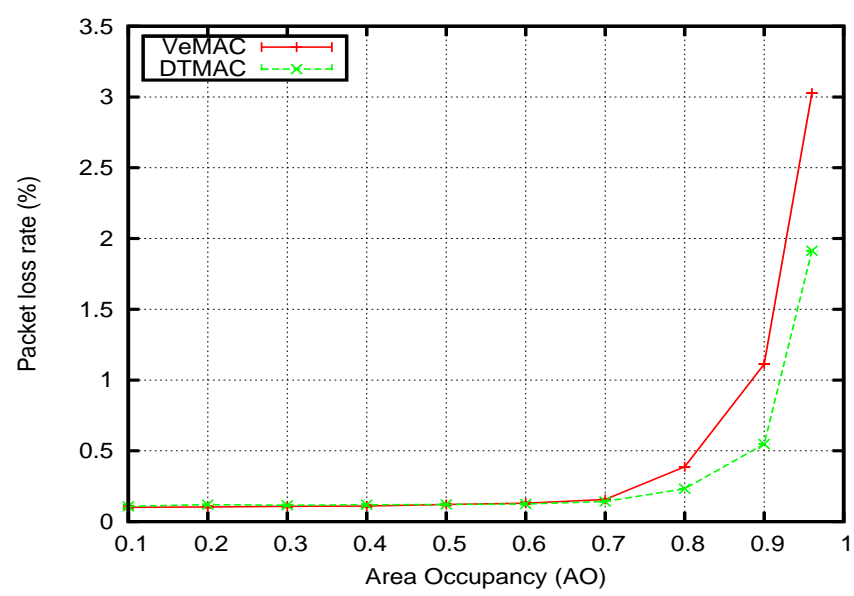

Fig. 8. The rate of packet loss under various traffic densities.

The packet loss rates of the two MAC protocols under consideration are shown in Figure 8. For a $A O \leq 0.7$, the DTMAC and VeMAC protocols have almost the same packet loss rate, while for a $A O>0.7$, DTMAC starts to perform better than VeMAC. It can be seen that our MAC protocol has the lowest packet loss rate, especially for a high $\mathrm{AO}$, due to its ability to handle the merging collision problem. For instance, at a $A O=0.96$, the VeMAC protocol shows approximately $58.23 \%$ higher rate of packet loss than the DTMAC protocol. The broadcast coverage rate is shown in Figure 9. It is clear that the two TDMA schemes achieved the same coverage ratio for low AO values. Note that for a high AO, DTMAC performs much better and the broadcast almost reached full 

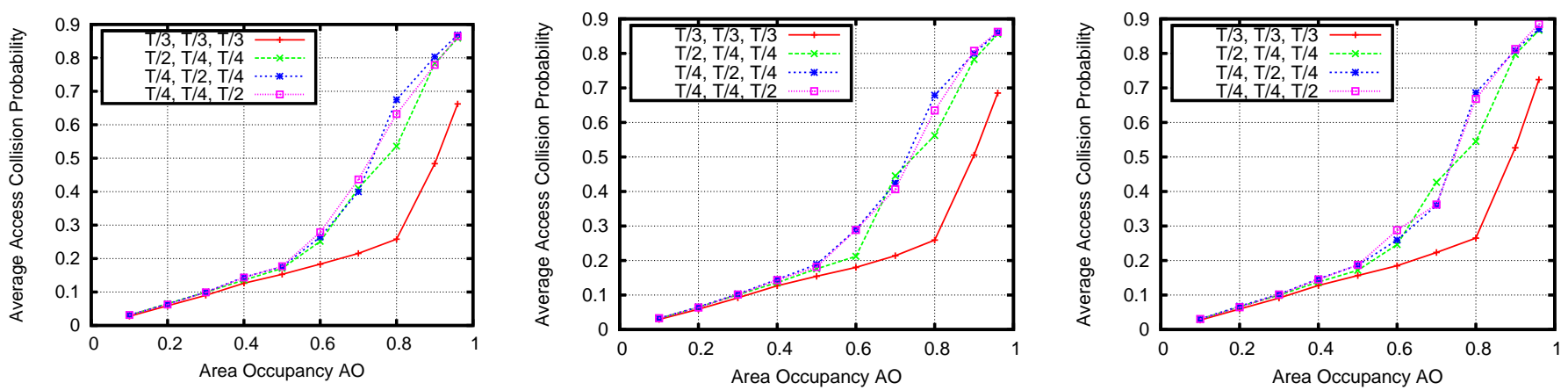

Fig. 5. The access collision probability for $\sigma$ equal to 20, 30 and $50 \mathrm{~km} / \mathrm{h}$, respectively.

coverage (i.e. $99.45 \%$ and $98.06 \%$ for $A O$ equal to 0.9 and 0.96 , respectively) .

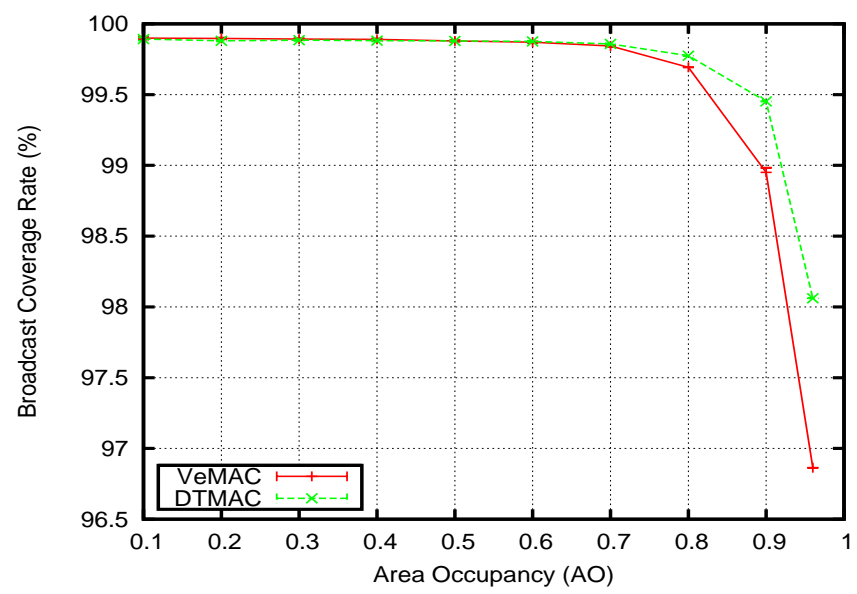

Fig. 9. The coverage broadcast ratio under various traffic densities.

\section{CONCLUSION}

Designing an efficient TDMA-based MAC protocol is an important issue in VANETs due to the rapid changes in network topology and the lack of infrastructure. In this paper, we propose a completely distributed and infrastructure-free TDMA scheduling scheme, named DTMAC which exploits the linear topology of VANETs. The ways that slots are allocated and reused between vehicles are designed to avoid collisions caused by the hidden node problem. The analytical model of the average access-collision probability is proposed. The simulation results show that, compared to VeMAC, DTMAC provides a smaller rate of access and merging collisions, which results in a significantly improved broadcast coverage.

In future work, DTMAC will be extended to support multichannel operation and to provide reliable broadcast on both the control and service channels. Moreover, we will carry out extensive simulations to compare it with the IEEE 802.11p standard. In addition, the future version of DTMAC will allow each vehicle to acquire more than one time slot per frame on the transmission channel.

\section{REFERENCES}

[1] M. Hadded, P. Muhlethaler, A. Laouiti, R. Zagrouba, and L. A. Saidane, "Tdma-based mac protocols for vehicular ad hoc networks a survey, qualitative analysis and open research issues," IEEE Communications Surveys Tutorials, vol. 17, no. 4, pp. 2461-2492, Jun. 2015.

[2] M. Hadded, R. Zagrouba, A. Laouiti, P. Muhlethaler, and L. A. Saidane, "An optimal strategy for collision-free slots allocations in vehicular adhoc networks," in Advances in Intelligent Systems and Computing, vol. 306, Kuala Lumpur, Malaysia, Jun. 2015, pp. 15-30.

[3] F. Ye, R. Yim, J. Zhang, and S. Roy, "Congestion control to achieve optimal broadcast efciency in vanets," in IEEE International Conference on Communications (ICC), Cape Town, South Africa, May 2010, pp. $1-5$.

[4] 802.11p-2010, IEEE standard for information technology - Telecommunications and information exchange between systems - local and metropolitan area networks - specific requirements part 11 : Wireless LAN medium access control (MAC) and physical layer (PHY) and physical layer (PHY) specifications amendment 6 : Wireless access in vehicular environments Std., 2010.

[5] R. Uzcátegui and G. Acosta-Marum, "Wave: A tutorial," IEEE Communications Magazine, vol. 47, no. 5, pp. 126-133, May 2009.

[6] N. Lu, Y. Ji, F. Liu, and X. Wang, "Dmmac: A dedicated multichannel mac protocol design for vanet with adaptive broadcasting," in IEEE Wireless Communications and Networking Conference (WCNC), Sydney, Australia, Apr. 2010, pp. 1-6.

[7] F. Borgonovo, A. Capone, M. Cesana, and L. Fratta, "Rr-aloha, a reliable r-aloha broadcast channel for ad-hoc inter-vehicle communication networks," in IEEE IFIP Annual Mediterranean Ad Hoc Networking Workshop (Med-Hoc-Net), Baia Chia, Italy, 2002.

[8] W. Zhuang, H. A. Omar, and L. Lio, "Vemac: A novel multichannel mac protocol for vehicular ad hoc networks," in IEEE Conference on Computer Communications Workshops (INFOCOM WKSHPS), Shanghai, China, Aug. 2011, p. 413418.

[9] F. Borgonovo, A. Capone, M. Cesana, and L. Fratta, "Adhoc mac: new mac architecture for ad hoc networks providing efficient and reliable point-to-point and broadcast services," Wireless Networks, vol. 10, no. 4, pp. 359-366, 2004.

[10] Y. Weidong, L. Pan, L. Yan, and Z. Hongsong, "Adaptive tdma slot assignment protocol for vehicular ad-hoc networks," Journal of China Universities of Posts and Telecommunications, vol. 20, no. 1, pp. 11-18, Feb. 2013.

[11] W. Ke, Y. Weidong, L. Pan, and Z. Hongsong, "A decentralized adaptive tdma scheduling strategy for vanet," in IEEE Wireless Communications and Networking Conference Workshops (WCNCW), Shanghai, China, Apr. 2013, pp. 216-221.

[12] D. N. M. Dang, H. N. Dang, V. Nguyen, Z. Htike, and C. S. Hong, "Her-mac: A hybrid efficient and reliable mac for vehicular ad hoc networks," in IEEE 28th International Conference on Advanced Information Networking and Applications (AINA), Victoria, Canada, May 2014, pp. 186-193.

[13] F. Borgonovo, L. Campelli, M. Cesana, and L. Fratta, "Impact of user mobility on the broadcast service efficiency of the adhoc mac protocol," in IEEE Vehicular Technology Conference, vol. 4, Dallas, TX, USA, May 2005, pp. 2310-2314.

[14] Vanetmobisim project home page. [Online]. Available: http://vanet.eurecom.fr/ 